\title{
The Early Days of EDS Development (and One Questionable Legacy)
}

\author{
Frederick Schamber ${ }^{1}$
}

1. 5095 Cherry Drive, Murrysville, Pennsylvania, USA

Sometimes one just happens to be at the right place at the right time, though it might not seem that way at the moment. It was like that for the author who, on the second day of his first permanent job, found himself being asked to divert from the task he had been hired for and undertake the development of software for an "x-ray analyzer" - a new and very vaguely-defined concept. This was in February of 1972, only four years after the publication of the seminal paper by Fitzgerald, Keil, and Heinrich [1] whose $50^{\text {th }}$ anniversary we are marking, and commercial EDS instrumentation was just appearing. This talk will offer observations on how developments were influenced by aspects of that early environment, and will highlight a particular vestige of that legacy which has a questionable place in the future of EDS.

An important fact about EDS technology is that it was built upon a pre-existing foundation of products developed for nuclear spectroscopy. Thus, when Fitzgerald set up to do his EDS experiment, he turned to a manufacturer of nuclear solid-state detectors (ORTEC) to fabricate a mechanical configuration that could be mounted on a microprobe, and Keil supplied a popular commercial multichannel analyzer (Nuclear Data) to collect the X-ray spectra [2]. As a consequence, the encouraging results obtained were soon being exploited by a group of manufacturers who were eager to expand the market for their own similar technology. This had important initial, and sometimes lasting, implications for EDS. For one thing, it established the model, still evident today, whereby the EDS functionality is considered a separate entity from the electron microscope, which is designed to accept standardized detector units available from multiple manufacturers. While it may be argued that this is optimal, it is nonetheless interesting to speculate how differently things might have turned out had a microscope manufacturer invented or first commercialized the EDS technology.

But it was not only nuclear-spectroscopy hardware which those early manufacturers brought to the embryonic EDS field, but also skills and attitudes. The author has described how his background in nuclear spectroscopy provided important insights employed in the development of the Filter-Fit algorithm for EDS spectrum analysis which is still widely used today [2]. More subtle is the way that the culture of research physicists may have influenced the way the technology evolved. Manufacturers servicing physics laboratories didn't see themselves as solving their customer's applications so much as giving them flexible components they could adapt to do it themselves. The presence of people with this mindset at the forefront of EDS development sometimes had "interesting" results. On the one hand, customers who purchased EDS instruments expecting turn-key solutions were often frustrated by how poorly some of those manufacturers understood their application. On the other hand, many of those early adopters of EDS technology were themselves researchers at heart and were delighted by the close working relationships that could be developed.

It's perhaps hard to appreciate now, but the purchase of an x-ray analyzer system was often heavily influenced by what else it could also be made to do. The birth of EDS coincided with the birth of "minicomputer" technology and since the x-ray analyzer not only housed what was probably the only computer in the laboratory, but also some "cutting edge" facilities such as a programmable CRT display, XYZ graphing outputs, and random-access data storage, it was a highly attractive platform for users to 
practice their own programming. The author is remembered by many old-timers as the creator of FLEXTRAN, a compact FORTRAN-like language which was employed for some very impressive user applications. In fact, the potent combination of a versatile computing platform and a close working relationship with innovative and skilled users accounts for why the author's company became for a time a major player in microprobe automation, digital imaging, and automated feature analysis.

However, one specific legacy of the nuclear spectroscopy heritage that is firmly rooted in the EDS vocabulary is now of questionable value -- the idea of dead-time. This concept originated with slow counting systems that were insensitive for a fixed period following each registered event. A simple formula was developed to correct counting rates and that is what is usually found in the general literature. Even though this simple idea of a fixed dead-time per event was not applicable to later detector systems that acquired energy spectra, it was still true that you could look at the system at any instant and say whether it was alive or dead. From this came the elegant idea of keeping track of the cumulative live-time, thus creating a scale factor by which spectra could be reliably compared. In effect, the live-time scale corrected for rate-dependent counting losses. And, as a convenience to the operator, it was customary to provide a meter displaying instantaneous fractional (percent) dead-time as a guide for achieving optimal throughput.

The concept of dead-time was further stretched when the digital pulse processor was introduced. In a literal sense, a modern EDS system operates with zero dead-time in that it collects data continuously. However, digital algorithms detect discrete events and decide whether or not a sufficiently precise measurement can be made. In the simplest cases, those decisions are made on simple ideas of event separation, and thus the idea of dead-time still makes some sense. However, as instruments become more sophisticated, simple ideas of "dead" or "live" lose their intuitive meaning for understanding the status of the system, and this will only get worse as the technology continues to evolve.

But it's not just that the traditional language of dead-time/live-time is becoming increasingly anachronistic: the nature of the problem has also changed. With the extremely high throughputs that can now be achieved with the combination of an SDD and digital pulse processor, the probability for unresolved multiple-event pileup has now become a critical issue, and this cannot be addressed by a live-time scale factor alone since it is now the shape of the spectrum that needs to be corrected. So what does "live-time" even mean when two spectra acquired for the same live-time can no longer be directly compared? And if some kind of intermediate processing must be done before live-time has a meaning, who standardizes that procedure? Or what should the venerable "dead-time meter" convey? Raw throughput? The probability of pileup? Something else? These are some of the challenging questions that the author believes will need to be addressed if we are to move forward without undue confusion.

But how is this best done? Shall we simply overlay yet another layer of abstraction on the already ambiguous dead-time/live-time terminology? If that is the choice, it means that we need to be scrupulous about distinguishing our new meanings and conventions from the old ones that appear in the literature. Or perhaps we will decide that it's finally time to "bury dead-time" and move forward with new terminology that better suits the reality of what we are now doing.

[1] R. Fitzgerald, K. Keil, \& K.F.J. Heinrich, Science 159 (1968), p. 528.

[2] K. Keil, R. Fitzgerald, \& K.F.J. Heinrich, Microscopy and Microanalysis 15 (2009), p. 476.

[3] F Schamber, Microscopy and Microanalysis 15 (2009), p. 491. 